Open Access

\title{
Toward green computing in wireless sensor networks: prediction-oriented distributed clustering for non-uniform node distribution
}

Ajay Sikandar ${ }^{1 *}$, Rajeev Agrawal ${ }^{1}$, Manoj Kumar Tyagi ${ }^{1}$, A. L. Narasimha Rao ${ }^{1}$, Mukesh Prasad $^{2}$ (D) and Muhammad Binsawad ${ }^{3}$

\author{
*Correspondence: ajay.sikender@ \\ glbitm.org \\ ${ }^{1}$ GL Bajaj Institute of Technology \\ and Management, Greater Noida, \\ India \\ Full list of author information is \\ available at the end of the article
}

\begin{abstract}
Recently, researchers and practitioners in wireless sensor networks (WSNs) are focusing on energy-oriented communication and computing considering nextgeneration smaller and tiny wireless devices. The tiny sensor-enabled devices will be used for the purpose of sensing, computing, and wireless communication. The hundreds/thousands of WSNs sensors are used to monitor specific activities and report events via wireless communication. The tiny sensor-enabled devices are powered by smaller batteries to work independently in distributed environments resulting in limited maximum lifetime of the network constituted by these devices. Considering the non-uniform distribution of sensor-enabled devices in the nextgeneration mobility centric WSNs environments, energy consumption is imbalanced among the different sensors in the overall network environments. Toward this end, in this paper, a cluster-oriented routing protocol termed as prediction-oriented distributed clustering (PODC) mechanism is proposed for WSNs focusing on nonuniform sensor distribution in the network. A network model is presented, while categorizing PODC mechanism in two activities including setting cluster of nodes and the activity in the steady state. Further cluster set up activity is described while categorizing in four subcategories. The proposed protocol is compared with individual sensor energy awareness and distributed networking mode of clustering (EADC) and scheduled sensor activity-based individual sensor energy awareness and distributed networking mode of clustering (SA-ADC). The metrics including the overall lifetime of the network and nodes individual energy consumption in realistic next-generation WSNs environments are considered in the experimental evaluation. The results attest the reduced energy consumption centric benefits of the proposed framework PODC as compared to the literature. Therefore, the framework will be more applicable for the smart product development in the next-generation WSNs environments.
\end{abstract}

Keywords: Green computing, Wireless sensor networks, Network prediction, Energy efficiency 


\section{Introduction}

The next-generation wireless sensor networks (WSNs) environment will be powered by a large number of tiny sensor-enabled devices that are designed to operate in the fields like smart building initiatives, automated location monitoring, indoor localizations, selfsustaining environment assessment and evaluation, smart home environment monitoring, intelligent systems for healthcare, future accurate forecasting of weather, fighting and controlling in battlefield, and intelligent operation of range of transport systems [1]. These sensor-enabled smart devices are used to effectively monitor various types of events which are purpose of interest in specified sensing fields and communicate their sensed observation data through wireless medium to the sinks, where computations are carried out and decisions are made [2].

However, these sensors are operated by coin-sized batteries and require human intervention to replace when required. Due to the limited and non-rechargeable batteries that are used as an energy or power source for the sensors, energy efficiency is a major concern, which has an effect on the network lifetime [3]. Hence, the major concern is to design a protocol which can reduce the energy consumption of individual nodes and thus support longer overall network lifetime for the next-generation WSNs environments. The sensors can be deployed in either planned or random fashion, depending on whether the sensing environment is known (planned) or not (random). Many research studies show that the clustering is used for energy constrain and maximizing network lifetime. In order to accomplish high energy efficiency and maximize network lifetime, sensors can be organized into cluster [4]. The cluster head election is an important aspect of WSNs, as designing a protocol for cluster-based communication, researchers face many problems due to complex nature of network topology to attain better energy efficiency [5]. The cluster head formation is based on the average energy consumption and residual energy of the neighboring sensors. To achieve the better energy efficiency, the sensor having no data packets to transfer to cluster head node can go into sleep mode, while sensor nodes with data can request to assign more time slot for transmitting the data. The cluster head receives the data packets from the cluster members, which forward data packets to sink node after data aggregation to omit redundant data, which in turn amortize the number of transmissions for saving the energy and bandwidth resources [6].

In WSNs, it is an essential aspect to balance the energy usage among sensors to improve network lifetime and reliability. The uniformly distributed nodes in a network balance the energy usage among nodes to prolong network lifetime, while non-uniform sensor distribution is considered less efficient [7]. Additionally, in a uniformly distributed sensor nodes, cluster head has uniform size which results in balanced energy consumption within the sensors, compared to the non-uniform sensor distribution, where energy usage of the sensors is more imbalanced [8]. The communication in WSNs can be intra-cluster or inter-cluster, in which the cluster head sensor uses time-division multiple access (TDMA) communication scheme to broadcast the schedule packet which contain the member information and slot number allocated to specific member sensor. The cluster head node allocates time slot to member sensors according to TDMA scheduling [9]. In low-energy adaptive clustering hierarchy (LEACH) protocol, the head of cluster node actually allocates the time slots for communication and provides information about timing and synchronization for all the sensors in the cluster, 
and the data packets are transmitted by only one node in the cluster for every allocated time slot for communication [10]. For a clustering-based communication, the sensorenabled nodes liaise with their head sensor node in the cluster. Similarly, the head of the cluster in the group liaises with only the base station (BS) node using a uniform time slot assignment scheme for same next-generation WSNs environment [11]. This way of communication is not appropriate for the nodes existing in different environment.

To overcome this situation, the head of the sensors in clustering can dynamically adjust the transmission slots to the member of the clustered sensors devices on demand. This should to done without using any uniform time slot assignment for the candidate sensors nodes for communication within the overall network. The sensors that fail to obtain timeslot are chosen to go to sleep mode for time and resource conservation and wait for next sessions to wake up and query cluster head for allotting time slots for transmitting data packets. The major contributions of the paper are as follows:

- A clustering of sensors centric green routing protocol, called prediction oriented distributed clustering (PODC), is proposed for non-uniform distributed nodes inside wireless sensor networks. Here, the cluster head election is based on the average energy consumption and average residual energy of the neighboring sensors. For energy consumption constraint, the proposed algorithm dynamically allocates the time slot using round robin method.

- A network model is presented, while categorizing PODC mechanism in two activities including setting up clusters of sensors and state of steady activity of sensors. Further, the cluster setting of sensors activity is described while categorizing in four subcategories including local information and information gathering activity, cluster head competition activity, sensor redundancy check and activation activity, and cluster design activity.

- Finally, the proposed protocol framework is assessed to comparatively evaluate the experimental performance with the state-of-the-art techniques such as energy awareness and distributed networking mode of clustering (EADC) and scheduled sensor activity-based individual sensor energy awareness and distributed networking mode of clustering (SA-EADC) considering the green computing-related metrics including the overall lifetime of the sensor network and individual energy consumption in sensor enabled devices in the next-generation WSNs environment.

The rest of the sections in the paper is organized as follows. In Section 2, a qualitative review on green computing techniques for the next-generation WSNs is carried out. In Section 3, the details of proposed green computing model for next-generation WSNs are presented. A discussion on simulation setup and analysis of experimental results are performed in Section 5 followed by the conclusion and future work in Section 5 .

\section{Related work}

Here, few energy-efficient cluster-based schemes are described along with scheduling algorithms for sensor nodes to exploit the redundant sensors and activity scheduling problems in WSNs. In [12], the authors suggested a cluster-based routing protocol, EADC along with routing algorithm for cluster-based communication for non- 
uniformly distributed nodes in wireless sensor networks. This algorithm makes cluster of even sizes considering competition range for balancing the energy usage among cluster nodes. In [13], the authors presented an improvement EADC as SA-EADC. It has utilized effective activity scheduling for enhancing the energy consumption performance of distributed clustering. The redundant sensors are selected to work by considering their residual energy. The proposed algorithm prevents unwanted sensing and data transmission unnecessary, which minimizes the overall energy usage of the sensors and increase life time of the network. In [14], the authors proposed local energy consumption prediction-based clustering protocol (LECP-CP). This protocol optimizes cluster radius size for minimizing the energy usage in the network. The proposed routing tree algorithm and clustering head election mechanism for communication between clusters considering prediction of local energy consumption ratio of the sensor nodes, shows an improvement in the lifespan of the network and energy requirement of the sensors.

In [9], the author presented a novel TDMA-based medium access control (MAC) protocol for energy conservation to improve the efficiency of transmitting the data for sensor nodes in cluster-based WSNs. In this protocol, the sensors which have no data to transmit, go to sleep mode while sensors with more data to transmit query the cluster head for allotting more time for transmitting the data. To achieve this, the round robin selection-based algorithm is implemented for the cluster head to minimize energy consumption and to improve latency. In [15], the author proposed a universal clustering-based routing (UCR) protocol for solving the hot spot problem in WSNs. It groups the sensors in to the clusters of unequal size. The UCR consists of an energy-efficient clustering algorithm for topology management, and a greedy geographic and energy aware routing protocol for communication between clusters.

In [16], a contiguous link scheduling algorithm has been proposed which is a novel interference free TDMA-based algorithm. For reducing the frequency of the state transmission, consecutive time slots are allotted to sensor nodes. The authors in [17] investigated an energy-efficient distributed clustering and scheduling algorithm for better performance of network. With this novel approach, the energy consumed by nonuniform sensor nodes is balanced for increasing the lifespan of the network. The literature review reveals that the clustering algorithms do not consider the efficient utilization of energy for improving the network lifetime particularly for large scale WSNs. In this paper, we consider this issue for intra and inter cluster communication with even and uneven node distribution.

A joint optimization problem, considering the network topology and unequal energy charge scheme for sensor nodes, has been formulated, and solved in order to extend the network lifetime and network coverage [18]. Authors proposed an energy-efficient traffic prioritization for medium access control protocol inside wireless body area networks. This protocol allocates slots with higher bandwidth and guards bands for reducing channel's interference which causes longer delay [19]. Author proposed wireless energy harvesting, wake-up radio scheme, and error control to enhance the performance of wireless sensor networks while reducing its carbon footprint. Author solved joint maximization problem and network life time. The packet loss and data utilization are incorporated to provide more realistic data loss and utilization model for the wireless sensor network system [20]. A traffic priority aware wireless access technique has 
been suggested focusing on reducing energy consumption and delay for green computing in wireless body networks [21]. Similarly, for green computing in cloud environment, energy centric computing algorithms has been suggested focusing on reducing service layer agreement violation during executing tasks on cloud servers [22]. Toward green computing-oriented communication, various techniques have been reviewed considering their strengths and weaknesses for underwater wireless network environment [23]. Similarly, another green computing-oriented critical investigation has been presented focusing on medium access control design [24]. These recent works majorly focuses on green computing and communication in wireless network environment which is the main theme of this research in the paper.

\section{Proposed green computing framework-PODC}

\subsection{System model}

The design issue for the proposed algorithm PODC is to consider the efficient utilization of energy for improving the network lifetime. Further, the considerations and assumptions of the algorithms are as follows: There are $\mathrm{n}$ sensors deployed in $\quad \times$

square field region in the network. The following assumptions and control messages are considered for deploying the network model (see Table 1). The symbols considered are described in Table 2.

- The sensors and sink node are stationary after deployment.

- The ( $n-$ number of sensors) are evenly distributed within the square field.

- The sensors are heterogeneous, w. r. to, energy and location information.

- The nodes continuously communicate their cluster head which in turn communicate to the base station.

- The nodes are distributed randomly within the network field.

- All sensors use power control approach to vary sensor transmission power.

Table 1 Nomenclature

\begin{tabular}{ll}
\hline Symbol & Description \\
\hline $\mathrm{n}$ & Number of sensors \\
$E_{T \times}(l, d)$ & Length of the region of interest \\
$\varphi$ & Transmission energy consumption for / bits and $d$ distance \\
$a$ and $\beta$ & Constant energy by electronic \\
$d_{(i, j)}$ & Amount of energy per bit dissipated in the transmitter amplifier \\
$E_{R x}(l)$ & distance between sensor i and sensor $j$ \\
$S_{i}$ & Receiving energy consumption for / bits \\
$E_{i a}$ & ith sensor \\
$E_{i a c}$ & Average residual energy of ith sensor \\
$t_{i}$ & Average energy consumption of ith sensor \\
$T_{2}$ & Waiting time \\
$T_{1}$ & Time duration for cluster head competition activity \\
$T_{3}$ & Time duration for information gathering \\
$T_{4}$ & Time duration for redundancy and activation \\
\hline
\end{tabular}


Table 2 Control messaging in PODC

\begin{tabular}{ll}
\hline Node_Message & Tuple (self_id, self_energy) \\
\hline Head_Message & Tuple (self_id) \\
Join_Message & Tuple (self_id, head_id) \\
Schedule_Message & Tuple (sechdule_order) \\
Route_Message & Tuple (self_id,self_energy, member_num, distt_BS) \\
Sleep_Message & Tuple (self_id, self_status) \\
\hline
\end{tabular}

The radio dissipation model adopted from [25] is used for the energy consumption in the transmission of sensors. The energy consumption is depending on the sum of constant electronic components for energy consumption and amplifier energy which is proportional to the receiver distance. The energy consumed by radio for transmitting the 1 bits message is described in Eq. (1).

$$
E_{T x}(l, d)= \begin{cases}l\left(\phi+\alpha d_{(i, j)}^{2}\right) & d_{(i, j)} \leq d_{0} \\ l\left(\phi+\beta d_{(i, j)}^{4}\right) & d_{(i, j)}>d_{0}\end{cases}
$$

where $\phi$ is the constant energy by electronic, $\alpha$ and $\beta$ are the amount of energy per bit dissipated in the transmitter amplifier, and $d_{(i, j)}$ is the distance between sensor $i$ and sensor $j$. The energy consumed by $E_{R x}(l)$ to receive the $l$ bits message is given by Eq. 2 .

$$
E_{R x}(\boldsymbol{l})=\boldsymbol{l} \times \boldsymbol{\phi}
$$

\subsection{PODC}

In this section, we describe the details of suggested protocol, PODC. The entire operation is accomplished in a round. The round is categorized into two activities: cluster set up activity and steady state activity. The cluster setup activity consists of four activities: local information and information gathering activity, cluster head competition activity, sensor redundancy check and activation activity, and cluster design activity. In the steady-state activity of transmission activity, it consists of $k$ sessions with fixed period. A session is divided into contention period, data transmission period, and idle period. Assuming there is $n$ number of non-cluster head required for $n$ number of slot of communication. Since the TDMA slot is not allocated to the member sensors, the non-cluster head sensors will have no data to transmit in the current round. More time slot is assigned if the sensor detects any sensor data.

Finally, the sensor which have not detected or observed any sensor data goes into sleep mode for reducing the energy usage. The cluster head election process is based on local observation function, which consists of ratio of average energy consumption and average residual energy of neighboring sensor to the residual energy and expected consumption of the sensor itself to balance the energy consumption among the cluster head.

\subsubsection{Cluster setup activity}

In the cluster setup activity, the entire process consists of five activities: the information gathering activity of time duration $T_{1}$, cluster head competition activity of time duration $T_{2}$, sensor redundancy check and activation activity of time duration $T_{3}$, and cluster formation activity of time duration $T_{4}$. 
3.2.1.1 Local observation and information gathering activity The activity duration is defined as $T_{1}$. In this activity, a node broadcasts the Node_Message to neighboring sensor with the transmission range $r$. The message has information: sensor id and residual energy. In response to the message, a node gets Node_Message from the neighboring sensors and it calculates the neighboring sensor distance through the receiver signal strength. Therefore, each sensor $s_{i}$ calculates the average residual energy $E_{i a}$ of the neighboring sensor and average energy consumption $E_{i a c}$ of the neighboring sensor. Every sensor $\mathrm{s}_{i}$ can be defined if local observation $(i)$ from their neighboring sensor by the Eq. 3 below.

$$
o(i)=\frac{E_{i a c}}{E_{i c}} * \frac{E_{i a}}{E_{i r}}
$$

where $E_{i c}$ represents the energy consumed by $s_{i}$ node and $E_{i}$ represents the residual energy of $s_{i}$ node. We describe the method to calculate the waiting time $t_{i}$ to broadcast Head_Message by

Eq. (4) below.

$$
t_{i}=o(i) * T_{2} * V_{r}
$$

Here, $T_{2}$ is the time duration for cluster head competition activity and $V_{r}$ represents a real value for uniformly distributed nodes, which reduces the probability of broadcasting Head Message at the same time by two nodes [26].

3.2.1.2 Cluster head competition activity After the completion of local observation and information gathering activity, the cluster head competition activity is started. In this activity, if a sensor $s_{i}$ do not receive any Head_Message during time $t_{i}$, the sensor itself broadcast a Head_Message to their neighboring sensors in competition radius $R_{c}$, or else it will not participate in competing for and becomes the plain sensor.

After the sensor $s_{i}$ broadcast Head_Message, the sensor will wait $2 * \Delta t_{i}$ time to make sure that there exists a sensor in the cluster range. Here, the time $\Delta t$ denotes the time interval that guarantees that all neighboring sensors can receive Head_Message.

3.2.1.3 Activation activity and checking for redundant sensors The activation activity and checking for redundant sensors begins at time duration $T_{3}$. In this activity nodes, redundancy and activity checking is performed by plain nodes. All sensor knows their status (i.e., plain or cluster head). All plain sensors in the network cooperatively participate without considering to which cluster they belong. To achieve complete coverage, each plain sensor executes crossing coverage for redundancy check [7]. The sensors with more battery power have higher chances to be active in each round. If the nodes identifies itself a redundant one, it reacts by setting time in proportional to the residual energy. If redundant node does not receives Sleep_Message within its set time limit, a Sleep_Message is broadcasted by the node for the range of $2 R_{s}$ along with current round status change as inactive and enters into sleeping mode of networking to reduce unnecessary energy consumption. 
3.2.1.4 Cluster formation activity The last activity of PODC algorithm begins at time duration $\mathrm{T}_{4}$. All the active plain sensor will choose the nearest cluster head according to the receiver's signal power. The plain sensors will transmit a Join_Message to the nearest cluster head. After receiving the Join_Message, each cluster head creates a sensor scheduling list based on the received "Join_Message." The cluster head also creates a Schedule_Message and send the message to the member nodes, which is used for identifying the transmission time of data for the nodes going into sleep mode for energy saving. Figure 1 below shows the cluster flowchart followed by the cluster setup algorithm.

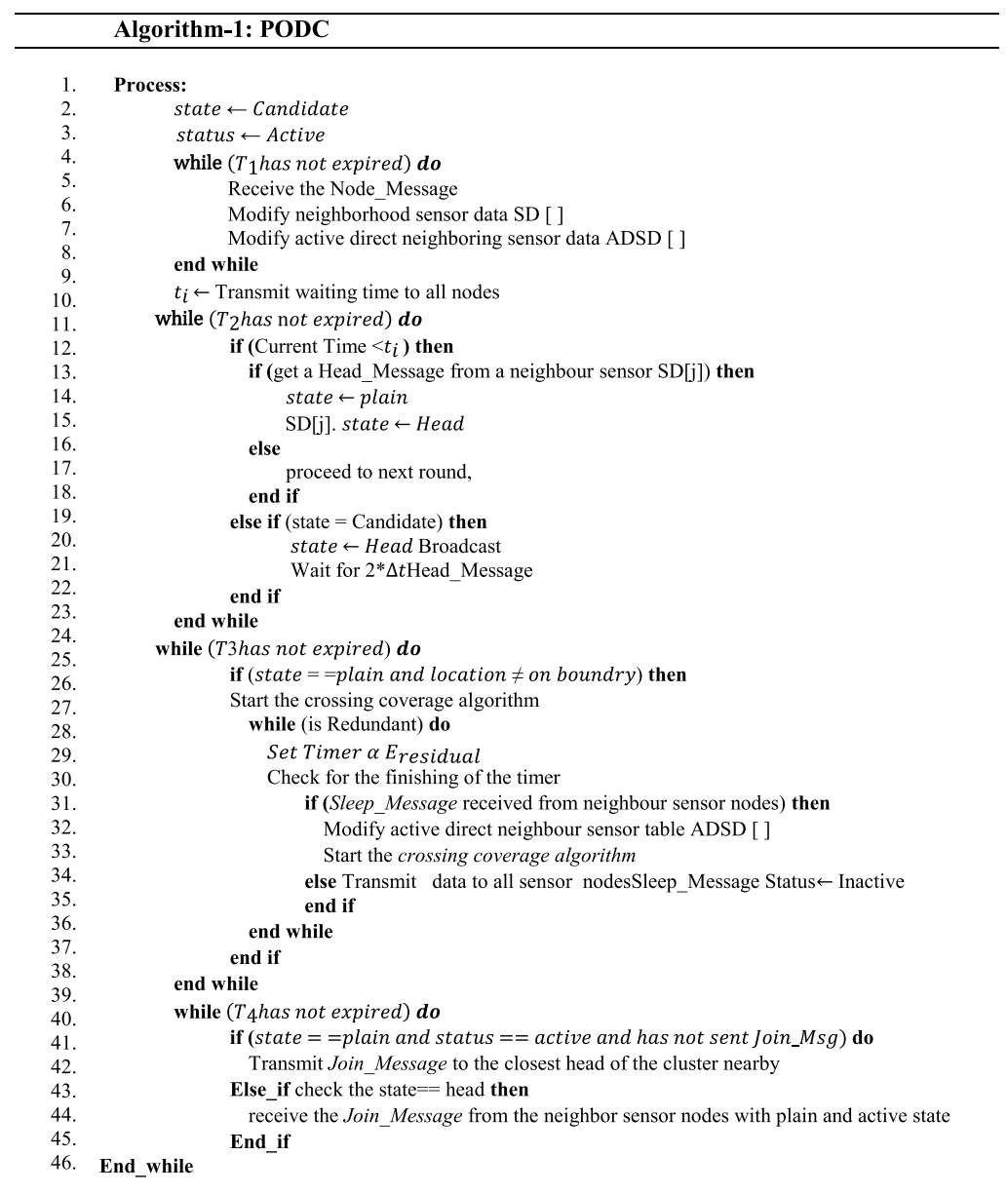

Once the cluster head is selected, the TDMA schedules transmission scheme (Schedule_Message) and starts a broadcast to the plain sensor within the range Ra and once the plain node is known, the TDMA schedule cluster formation activity is completed and the next activity steady-state activity (data transmission) begins.

\subsubsection{Steady-state activity}

The steady-state activity is consisting of $K$ sessions for a round and a session has contention period, advertisement period, idle period, and data transmission period. In each session, data transmission period is variable; however, the data transmission period along with idle period is always fixed; also, all the sensors become active during this 


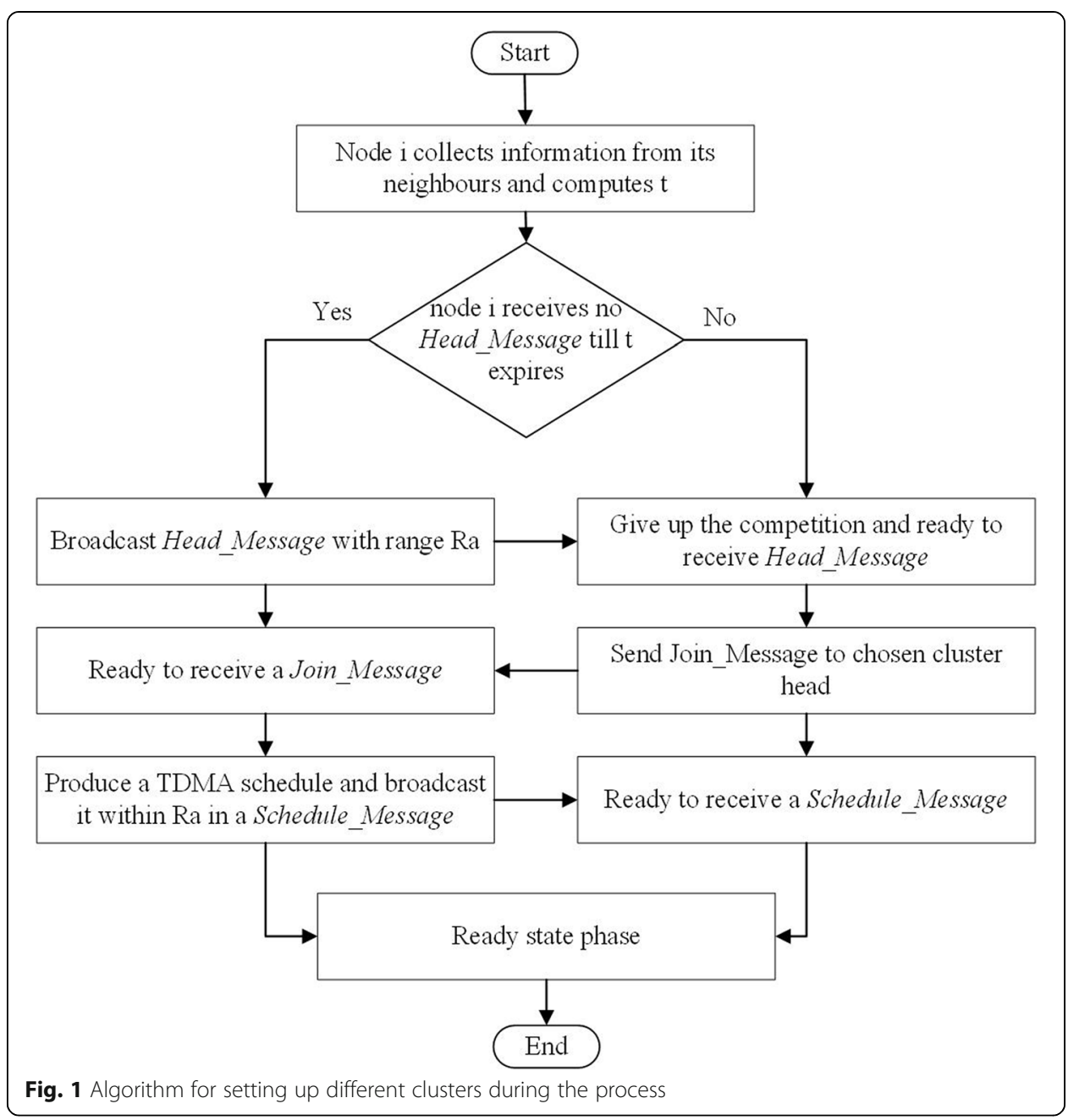

contention period. The contention period follows TDMA time slot schedule and each sensor is allocated a specific time slot to transmit 20-bytes of control message. In this slot time, the sensors will transmit the data if it had any or else it will keep the slot time empty. Figure 2 depicts the design of single round.

The transmission schedule is implemented using round robin-based method for assigning the slot for the observed data from a source sensor. The sensors with no data change to sleeping mode. If the sensors have observed data from environment, it will request to cluster head for allotting more time slots for transmitting the data. Here, the energy can be conserved by all sensors if they have no data to send and on the other hand, more time slot is allocated to the sensors that have more data to send. In this paper, a scheduling algorithm has been designed which allots the time slots for the nodes using round robin approach and maintain fairness. Assuming that the number of slots required is equal to the number of plain sensors within the cluster, considering some sensors have no data to send while other sensor have more data to send. In this situation, the sensors with more data will be given priority and allocated more time slot for transmission in round robin fashion so that fair allocation of slots can be possible.

As depicted in Fig. 3 below, the sensor S1 uses two slots for transmitting the observed data from environment; S2 sensor uses two slots for transmitting observed data 


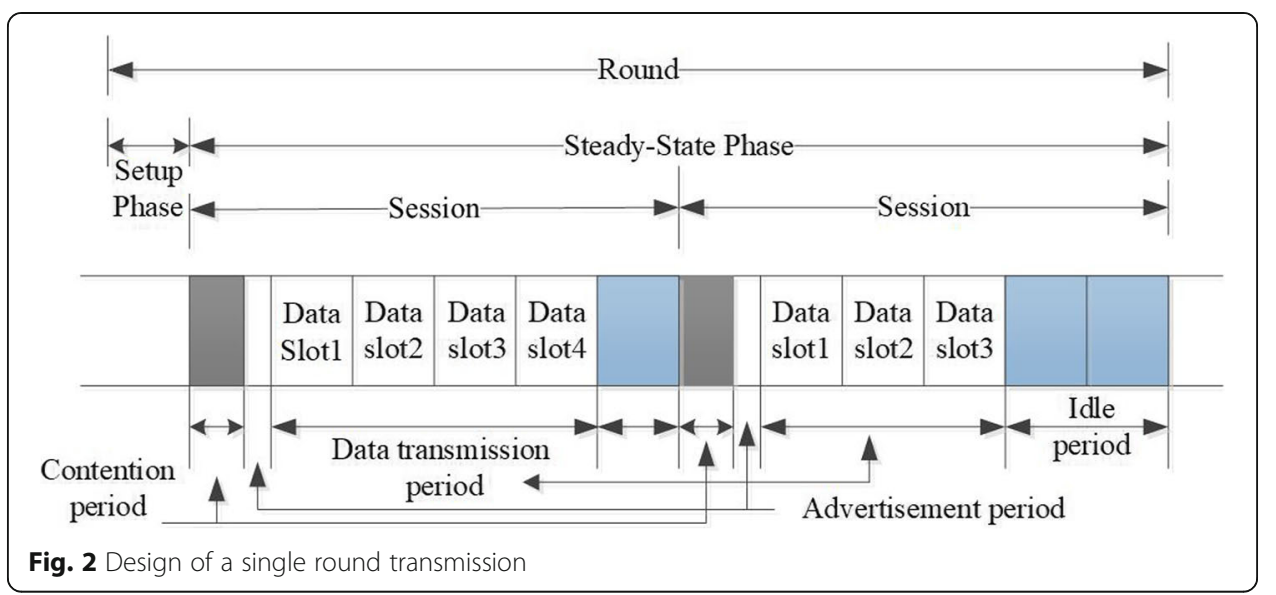

from environment; S3 and S4 has no sensed data from environment; S5 uses one time slot for transmitting observed data from environment; S6 and S7 needs no sensed data from environment; S8 uses three time slots for transmitting data from environment; S9 needs no sensed data from environment and S10 uses two time slot for transmitting data from environment is given by slot allotment algorithm below.

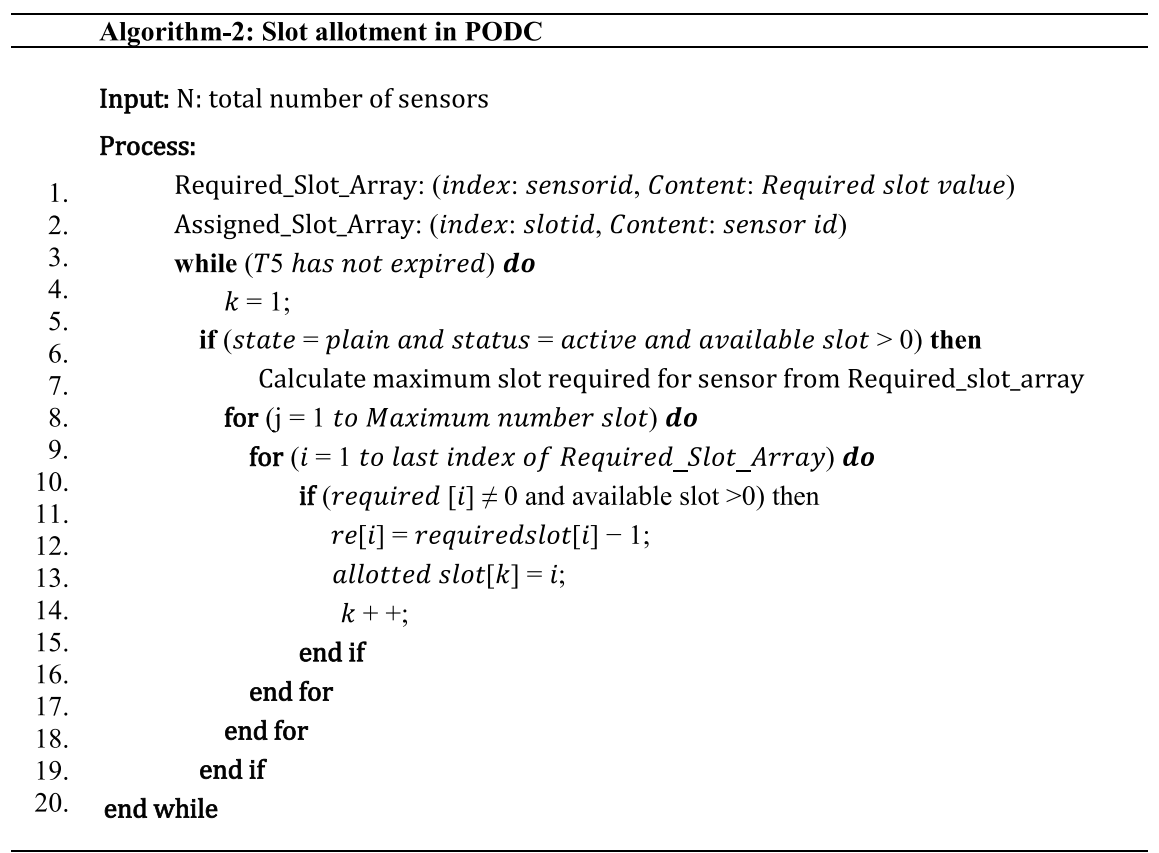

\section{Results and discussion}

In this section, the performance evaluation of the proposed protocol PODC is presented and is compared with SA-EADC and EADC protocols. For validation in terms of performance of the PODC, it is simulated over the Matlab with clustered wireless network for the area of dimension $200 * 200 \mathrm{~m}^{2}$. Some results are omitted which are caused by the signal collision and interference on wireless channels. The parameters used for simulation is provided in Table 3. For the simulation, two test contexts are considered and they are as follows:

(i) Context 1: 100 sensors are randomly distributed over the area of 200*200 $\mathrm{m}^{2}$. 


\begin{tabular}{|c|c|c|c|c|c|c|c|c|c|c|}
\hline & 1 & 2 & 3 & 4 & 5 & 6 & 7 & 8 & 9 & 10 \\
\hline S1 & & & & & & & & & & \\
\hline S2 & & & & & & & & & & \\
\hline S3 & & & & & & & & & & \\
\hline S4 & & & & & & & & & & \\
\hline S5 & & & & & & & & & & \\
\hline S6 & & & & & & & & & & \\
\hline S7 & & & & & & & & & & \\
\hline S8 & & & & & & & & & & \\
\hline S9 & & & & & & & & & & \\
\hline $\mathrm{S} 10$ & & & & & & & & & & \\
\hline
\end{tabular}

Fig. 3 TDMA slot allotment for cluster member sensor sensors

(ii) Context 2: 100 sensors are non-uniformly deployed over the area $200 * 200 \mathrm{~m}^{2}$.

The aforementioned contexts are used for validating the applicability of PODC protocol to save the energy and for increasing the lifespan of the network.

\subsection{Network lifetime}

Figure 4 depicts the network lifetime per round versus cluster range for context 1 . The network life time is expressed when all the sensors are alive. The cluster range is set up between 100 and $220 \mathrm{~m}$ for both contexts. For precisely studying the impact of range of sensing on the life time of the overall networking configuration, the sensing range is set to between 20 and $25 \mathrm{~m}$ for both contexts. The results obtained in the simulation shows that the network life time per round increases as compared to EADC and SA-

Table 3 Simulation parameters

\begin{tabular}{ll}
\hline Number of sensors & 100 \\
\hline Sink location & $(250,100)$ \\
Sensor field & $100 \times 100$ \\
Initial energy of sensors & $1-3 \mathrm{~J}$ \\
$\varphi$ & $50 \mathrm{nj} / \mathrm{bit}$ \\
$a$ & $10 / \mathrm{pj} / \mathrm{bit} / \mathrm{m} 2$ \\
$\beta$ & $0.0013 \mathrm{pj} / \mathrm{bit} / \mathrm{m} 4$ \\
Data packet size & $5000 \mathrm{bits}$ \\
Control packet size & $500 \mathrm{bits}$ \\
Sleep power & $15 \mu \mathrm{W}$ \\
$\mathrm{E}_{\text {sen }}$ & $0 \mathrm{~J} / \mathrm{bit}$ \\
$\mathrm{E}_{\text {com }}$ & $5 \mathrm{nj} /(\mathrm{bit} \mathrm{signal})$ \\
\hline
\end{tabular}




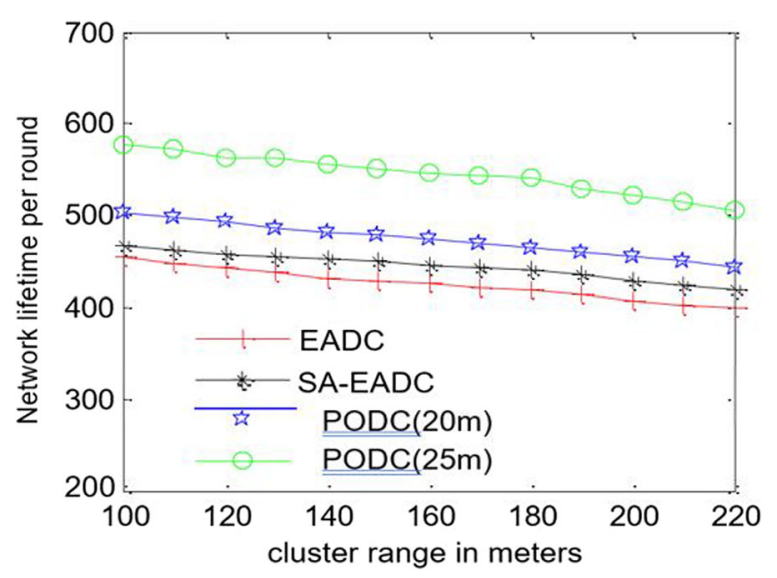

Fig. 4 Network life time (context 1)

EADC. It can be also observed that the increase in the sensing range also increases the network life time of the system.

Figure 4 (context 1 ) presents the slightly longer network life time per round for the proposal as compared to the existing literature considered with $20 \mathrm{~m}$ of range for sensing. However, significant improvement in network life time is observed by increasing the sensing range to $25 \mathrm{~m}$. The network life time is improved by $7.5 \%$ and $11 \%$ for the sensing range of $20 \mathrm{~m}$ as compared with the SA-EADC and EADC respectively. Also, the network lifetime improvement is achieved for sensing range $25 \mathrm{~m}$ is $23 \%$ and $27 \%$ as compared with the SA-EADC and EADC protocols respectively.

In Fig. 5 (context 2), for sensing range $20 \mathrm{~m}$, network lifetime is improved by $7.6 \%$ and $10.3 \%$ as compared to SA-EADC and EADC respectively. Also, an improvement of $23 \%$ and $26 \%$ is noticed as compared with the protocol SA-EADC and EADC respectively for sensing range of $25 \mathrm{~m}$. It can be observed that the performance of the proposed framework PODC has durable network lifetime per round as compared to the state-of-the-art techniques considered including EADC and SA-EADC. In particular, with 100 -m cluster range, the network lifetime is 500 and 580 rounds, whereas it is 460 and 470 rounds for EADC and SA-EADC respectively. Similarly, the network lifetime is

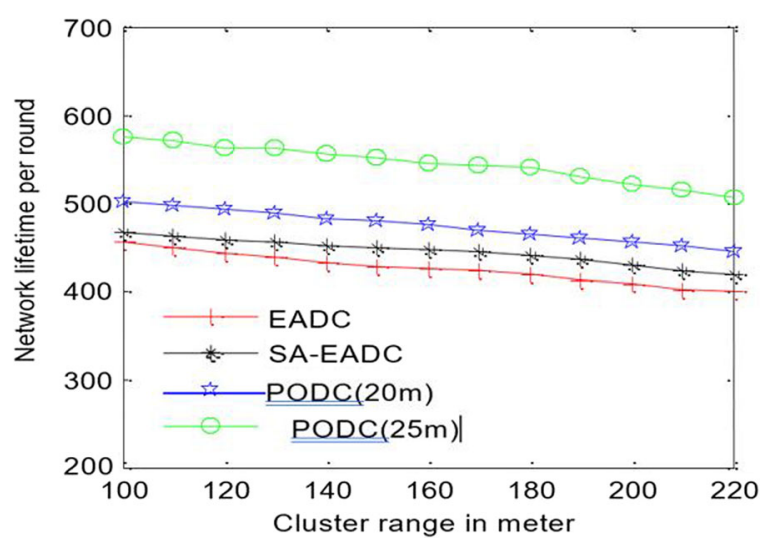

Fig. 5 Network life time (context 2) 
480 and 540 rounds for PODC with 200-m cluster range, whereas it is 410 and 420 rounds for EADC and SA-EADC.

\subsection{Energy consumption}

Figures 6 and 7 show the average energy consumption by sensor nodes in constructing the network topology, transmitting data, and ideal stage energy wastage in sleeping state in each networking round. This is measured in terms of dissipated energy in joules versus cluster range in meters. The result obtained from the simulation shows that PODC sustains for increasing the lifetime of the network w. r. to EADC and SA-EADC. The average energy requirement for the sensors for every round increases slowly with the increase of the cluster size and there is decrement in the energy consumption per round is also observed when the sensing range of the protocol is increased.

In Fig. 6 (context 1), it can be seen the decrement in energy consumption by $15 \%$ and $24 \%$ for the sensing range of $20 \mathrm{~m}$ as compared with the SA-EADC and EADC, respectively. Also, a more decrement in energy consumption is noticed around $24 \%$ and $32 \%$ at a range of $25 \mathrm{~m}$ by PODC compared to SA-EADC and EADC protocol. It can be noticed that the performance of the proposed framework PODC is better in terms of lower dissipated energy in comparison with those of the state-of-the-art techniques considered including EADC and SA-EADC. For example, with 100-m cluster range, the dissipated energy is $0.27 \mathrm{~J}$ and $0.28 \mathrm{~J}$ for PODC, whereas it is $0.34 \mathrm{~J}$ and 0.37 rounds for EADC and SA-EADC respectively. Similarly, the dissipated energy reaches to $0.30 \mathrm{~J}$ and $0.31 \mathrm{~J}$ for PODC with 200-m cluster range, whereas it is $0.35 \mathrm{~J}$ and $0.48 \mathrm{~J}$ for EADC and SA-EADC.

In Fig. 7 (context 2), for the sensing of range $20 \mathrm{~m}$, decrement in energy consumption of $15 \%$ and $24 \%$ is observed as compared to SA-EADC and EADC, respectively. It is also observed that the decrement in energy consumption of $24 \%$ and $32 \%$ for PODC at sensing range of $25 \mathrm{~m}$ as compared with the SA-EADC and EADC protocols.

\subsection{Standard deviation of residual energy}

In this section, standard deviation of residual energy (SDE) of sensor in each round is studied focusing on construction of network topology, transmission of data, and

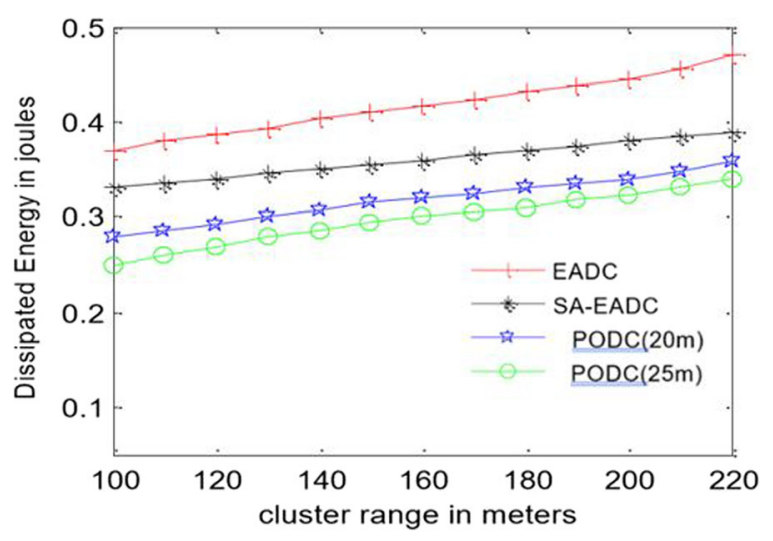

Fig. 6 Average energy dissipation per round (context 1) 


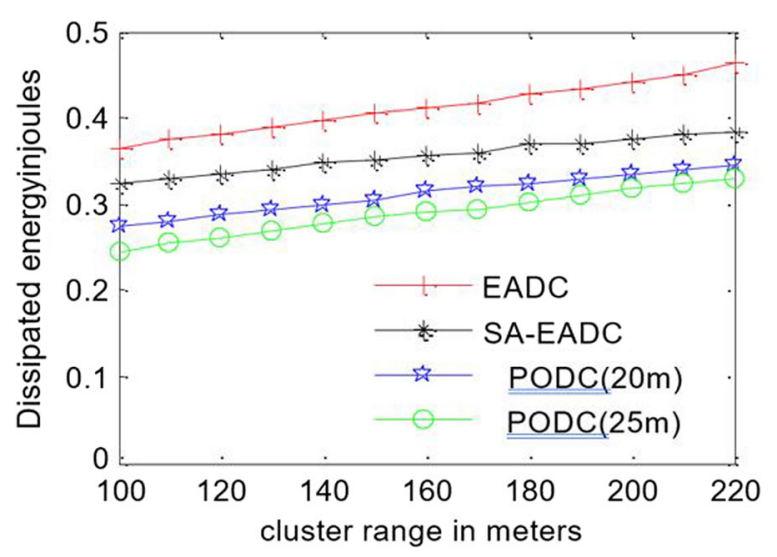

Fig. 7 Comparison of energy dissipation in each round considering context 2

sleeping sensor node mode. Figures 8 and 9 illustrate standard deviation of residual energy versus simulation time. The comparison of standard deviation of energy at the end of simulation between PODC and state of art techniques is presented. The results obtained in the simulation show that the standard deviation of energy of PODC is lesser as compared with the SA-EADC and EADC.

In context 1 when $20 \mathrm{~m}$ is considered as sensing range, standard deviation of residual energy in the case of PODC is 0.1 and 0.01 SDE higher than state of the art protocol SA-EADC and EADC at the end of simulation. Because for PODC, the standard deviation of residual energy (SDE) is $0.34 \mathrm{SDE}$, and for EADC and SA-EADC are 0.44 and 0.35 SDE.

In context 2 , when the sensing range is set to $25 \mathrm{~m}$, the standard deviation of residual energy (SDE) in the case of PODC is 0.1 and 0.01 SDE higher than the state-of-the-art protocol SA-EADC and EADC at the end of simulation. Because for PODC, the standard deviation of residual energy is $0.32 \mathrm{SDE}$, and for EADC and SA-EADC are 0.42 and $0.33 \mathrm{SDE}$. It can be observed that the performance of the proposed framework PODC is better in terms of standard deviation of energy in comparison with those of the state-

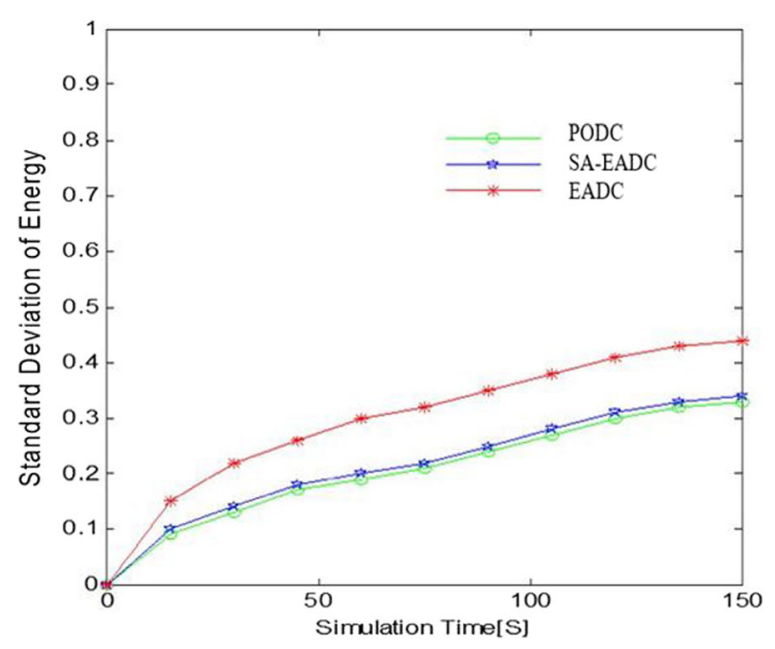

Fig. 8 Standard deviation of residual energy (context 1) 


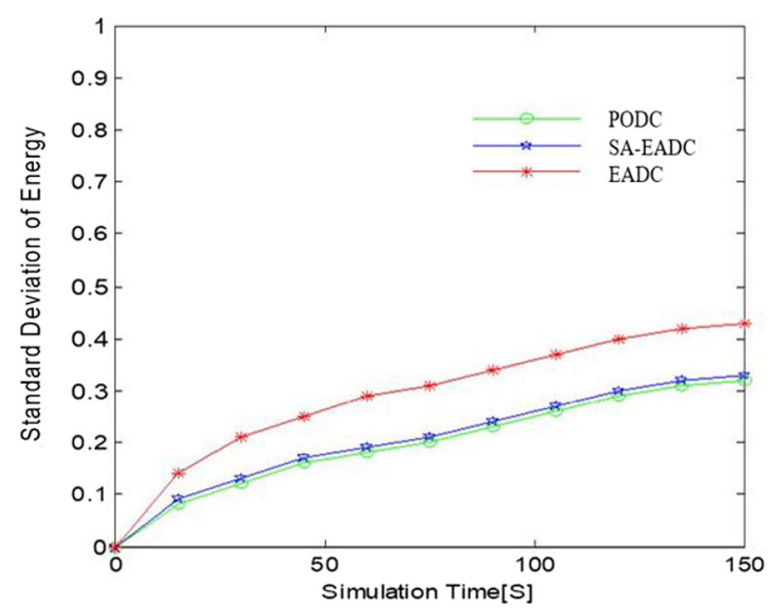

Fig. 9 Standard deviation of residual energy (context 2)

of-the-art techniques considered including EADC and SA-EADC. For example, with 50 -s simulation time, the standard deviation of energy is approximately 0.15 for PODC, whereas it is 0.16 and 0.22 for SA-EADC and EADC respectively. Similarly, the standard deviation of energy reaches to 0.32 for PODC with 150-s simulation time, whereas it is 0.33 and 0.42 for SA-EADC and EADC.

\section{Conclusion}

In this paper, a novel clustering protocol, PODC, is suggested for WSNs, in which a new cluster head strategy for non-uniformly distributed nodes and network lifespan is introduced. Mathematical models are derived for scientific understanding of consumption of energy by sensors and the lifetime of overall wireless sensor network. The simulation results and analysis proves that overall energy consumption in sensors can be reduced by putting the sensors, which plays no part in transmitting the data into a sleep mode and given more priority and bigger time slots for the sensors who play important role in data transmission. The result shows the improvement in network lifetime and energy dissipation among sensor nodes. The suggested protocol is compared with SA-EADC and EADC, in terms of network lifetime and average energy dissipation among sensors with multiple contexts and it is observed that the PODC performs far better than them. Therefore, it can be safely concluded that PODC improves the network life span while maintaining original sensing coverage level for the network.

\section{Abbreviations}

WSNs: Wireless sensor networks; PODC: Prediction oriented distributed clustering; EADC: Energy awareness and distributed networking mode of clustering; SA-EADC: Scheduled sensor activity-based individual sensor energy awareness and Distributed networking mode of clustering; TDMA: Time-division multiple access; LEACH: Low-energy adaptive clustering hierarchy; LECP-CP: Local energy consumption prediction based clustering protocol; MAC: Medium access control; UCR: Universal clustering based routing; SDE: Standard deviation of residual energy

\section{Authors' contributions}

AS has modeled and executed the research. RA and MKT have supervised the research. AS has designed the experimental testing and data visualization. ANR and MP have validated mathematical design and testing. MB has enhanced the quality of the research by their valuable comments and suggestions in data analysis and discussion. AS has written the paper and MP has improved the technical writing of the paper with comments and suggestions. The authors read and approved the final manuscript. 


\section{Funding}

The research is funded by the King Abdulaziz University, Saudi Arabia and supported by the University of Technology, Sydney, Australia and GL Bajaj Institute of Technology and Management, Greater Noida India.

\section{Availability of data and materials}

The experimental data and associated settings will be made available to researchers and practitioners on individual requests with the restrictions that it will be used for further investigation with collaborative research progress only.

\section{Competing interest}

It is declared that there is no competing interest among authors.

\section{Author details}

${ }^{1}$ GL Bajaj Institute of Technology and Management, Greater Noida, India. ${ }^{2}$ School of Computer Science, FEIT, University of Technology Sydney, Sydney, Australia. ${ }^{3}$ Department of Computer Information Systems, Faculty of Computing and Information Technology, King Abdulaziz University, Jeddah, Saudi Arabia.

Received: 13 April 2020 Accepted: 14 August 2020

Published online: 22 September 2020

\section{References}

1. O. Kaiwartya, A. H. Abdullah, Y. Cao, A. Altameem,M. Prasad, C. T. Lin and X. Liu, "Internet of vehicles: motivation, layered architecture, network model, challenges, and future aspects," IEEE Access, 4, pp.5356-5373, 2016.

2. O. Kaiwartya, A.H. Abdullah, Y. Cao, J. Lloret, S. Kumar, R.R. Shah, M. Prasad, S. Prakash, Virtualization in wireless sensor networks: fault tolerant embedding for internet of things. IEEE Internet of Things Journal 5(2), 571-580 (2018)

3. A. Khatri, S. Kumar, O. Kaiwartya, A.H. Abdullah, Green computing for wireless sensor networks: Optimization and Huffman coding approach. Peer-to-Peer Networking and Applications 10(3), 592-609 (2017)

4. O. Kaiwartya, S. Kumar, A.H. Abdullah, Analytical model of deployment methods for application of sensors in non-hostile environment. Wireless Personal Communications 97(1), 1517-1536 (2017)

5. A. Khatri, S. Kumar, O. Kaiwartya, N. Aslam, N. Meena and A. H. Abdullah, "Towards green computing in wireless sensor networks: Controlled mobility-aided balanced tree approach," International Journal of Communication Systems, 31(7), p. e3463 (2018)

6. A. Khatri, S. Kumar, O. Kaiwartya, and A. H. Abdullah, "Optimizing energy consumption and inequality in wireless sensor networks using NSGA-II," In Communication and Computing Systems: Proceedings of the International Conference on Communication and Computing Systems (ICCCS 2016), Gurgaon, India, 9-11 September, 2016 (p. 367). CRC Press.

7. J. Yu, Y. Qi, G. Wang, X. Gu, A cluster-based routing protocol for wireless sensor networks with nonuniform sensor distribution. AEU—International Journal of Electronics and Communications 66(1), 54-61 (2012)

8. H. Shin, S. Moh, and I. Chung, "A balanced clustering algorithm for non-uniformly deployed sensor networks," in Proceedings of the IEEE 9th International Conference on Dependable, Autonomic and Secure Computing (DASC '11), pp. 343-350, Sydney, Australia, December 2011.

9. T.H. Hsu, P.Y. Yen, Adaptive time division multiple access based medium access control protocol for energy conserving and data transmission in wireless sensor networks. IET Communication 5(18), 2662-2672 (2011)

10. J. Hu, Y. L. Dou, " A time-based cluster-head selection algorithm for LEACH," IEEE, 2008.

11. S.C. Ergen, P. Varaiya, TDMA scheduling algorithms for wireless sensor networks. Wireless Networks 16(4), 985-997 (2010)

12. J. Yu, Y. Qi, G. Wang, X. Gu, A Cluster-based routing protocol for wireless sensor networks with non-uniform sensor distribution. International Journal of Electronic and Communication, Elsevier 66(1), 54-61 (2012)

13. N. Nokhanji, Z. M. Hanapi, S. Subramaniam, M. A. Mohamed, A scheduled activity energy aware distributed clustering algorithm for wireless sensor networks with nonuniform sensor distribution," International Journal od Distributed Sensor Networks, Hindawi, Vol. 2014, 2014, PP.133-139.

14. J. Yu, L. Feng, L. Jia, X. Gu, D. Yu, A local energy consumption prediction based clustering protocol for wireless sensor networks. SENSORS 14(8), 23017-23040 (2014)

15. G. Chen, C. Li, M. Ye, J. Wu, An unequal cluster-based routing protocol in wireless sensor network. Wireless Networks, Springer 15(2), 193-207 (2009)

16. J. Ma, W. Lou, Y. Wu, M. Li, G. Chen, "Energy efficient TDMA sleep scheduling in TDMA in wireless sensor networks," IEEE Infocom, 2009, pp.630-638

17. J. Peng, T. Liu, H. Li, B. Guo "Energy-efficient prediction clustering algorithm multilevel heterogeneous wireless sensor network," International Journal of Distributed Sensor Networks, Hindawi, Vol.2013, 2013pp.1-7

18. A. Xenakis, F. Foukalas, G. Stamoulis, I. Katsavounidis, "Topology control with coverage and lifetime optimization of wireless sensor networks with unequal energy distribution," Computer and Electrical Engineering, Vol. 000(2017), Elsevier, pp. 1-18

19. F. Ullah, A. H. Abdullah, O. Kaiwartya, J. Lioret, M. M. Arshad, "EETP-MAC: energy efficient traffic prioritization for medium access control in wireless body area networks," Telecommunication Systems, Springer, 2017, pp.1 23.

20. C. Mahapatra,Z. Sheng, P. Kamalinejad, V. Leung, S. Mirabbasi, "Optimal power control in green wireless sensor network with wireless energy harvesting, wake-up radio and transmission control" IEEE access, Vol. 5,2017,pp. 501-518

21. F. Ullah, A.H. Abdullah, O. Kaiwartya, Y. Cao, TraPy-MAC: traffic priority aware medium access control protocol for wireless body area network. Journal of medical systems 41(6), 93 (2017)

22. R. Yadav, W. Zhang, O. Kaiwartya, P.R. Singh, I.A. Elgendy, Y.C. Tian, Adaptive energy-aware algorithms for minimizing energy consumption and SLA violation in cloud computing. IEEE Access 6, 55923-55936 (2018)

23. A. Khasawneh, M.S.B.A. Latiff, O. Kaiwartya, H. Chizari, Next forwarding node selection in underwater wireless sensor networks (UWSNs): Techniques and challenges. Information 8(1), 3 (2017) 
24. F. Ullah, A.H. Abdullah, O. Kaiwartya, S. Kumar, M.M. Arshad, Medium access control (MAC) for wireless body area network (WBAN): Superframe structure, multiple access technique, taxonomy, and challenges. Human-centric Computing and Information Sciences 7(1), 34 (2017)

25. W. B Heinzelman, A. P. Chandrakasan, H. Balakrishanan, "An application specific protocol architecture for wireless microsensor networks," IEEE Transaction on Wireless Communication, Vol. 1, No. 4, 2002, pp.660-670.

26. I. Akyildz, W. Su, Y. Sankarasubramaniam, E. Cayirci, A survey on sensor network. IEEE Communication Magzine 40(8), 102-114 (2000)

\section{Publisher's Note}

Springer Nature remains neutral with regard to jurisdictional claims in published maps and institutional affiliations.

Submit your manuscript to a SpringerOpen ${ }^{\circ}$ journal and benefit from:

- Convenient online submission

- Rigorous peer review

- Open access: articles freely available online

- High visibility within the field

- Retaining the copyright to your article 